\title{
Kinematics effect on honeycomb sandwich beams vibration
}

\author{
I. Chenini ${ }^{\mathrm{a}}$, R. Nasri, C. Mrad And Y. Abdelli \\ Applied Mechanics and Engineering Research Laboratory (LR-MAI), National Engineers School of Tunis, \\ University of Tunis El Manar, Tunisia
}

Received 1 May 2015, Accepted 23 August 2016

\begin{abstract}
Composite structures are used in aerospace, aeronautics, and automotive industries, especially composite sandwich panels which are exposed to vibration, noise, and unusual loads. They are often considered to support vibrations due to their important resistance to fatigue. The aim of this work is to study the influence of displacement fields on the vibration of honeycomb sandwich beams, considering two materials: Nomex paper and Aluminum alloy. For this purpose, the minimum energy method is used to derive the natural frequencies and modal shapes equations of a cantilever honeycomb sandwich beam. The kinetic and potential energies of skins are derived using the classical laminate theory, and several polynomial displacement fields are tested. The effect of core rotational inertia and bending energy on the sandwich beam vibration is also studied. The modal shapes are then examined. Besides, an experimental study is done on the cantilever honeycomb sandwich beam, using an impact hammer for excitation and a laser vibrometer for measurement. The natural frequencies are then obtained by modal analysis. In addition, a numerical simulation is conducted on the cantilever honeycomb sandwich beam to complete this work, and the natural frequencies obtained analytically, numerically, and experimentally are compared. Future steps to enhance this work are finally advanced.
\end{abstract}

Key words: Sandwich beam / bending vibration / displacement fields / natural frequencies / modal shapes

\section{Introduction}

Energy methods are among the most important methods to study the vibration of beams. The problem with these methods is that they require the introduction of a field of kinematically admissible displacement or statically admissible stress or both simultaneously.

The method accuracy will therefore depend on a righteous choice of the field. Several displacement fields are introduced in the literature, but the experimental results presented do not help in choosing an adequate displacement field.

Mindlin [1] proposed a model to study the transverse shear of a thick isotropic sandwich plate, it is known as the FSDT theory (First-order Shear Deformation Plate Theory).

Yarlagadda et al. [2] developed an analytical method based on Rayleigh-Ritz theory in order to determine the influence of fiber orientation, temperature, and thickness on natural frequencies, modal shapes, and modal damping of the composite plate.

Wang [3] compared the Classical Laminated Plate Theory (CLPT) with the Mindlin's theory (FSDT) by

\footnotetext{
${ }^{a}$ Corresponding author: chenini.idris@yahoo.fr
}

studying the bending vibrations of a rectangular skin and core isotropic sandwich panel.

Meunier and Shenoi [4] introduced the mechanical properties of the components of the sandwich plate, in the analytical equations of elastic and viscoelastic models, and the frequencies obtained highlighted the properties effect.

Soula et al. [5] studied the influence of the kinematics on laminated plates vibration. They used three plate theories: Kirchhoff, Mindlin, and Reddy; to estimate the natural frequencies and modal damping of symmetric and asymmetric plates.

Maheri and Adams [6] presented an experimental study in order to obtain the loss factor for honeycomb sandwich beams, considering fiber orientation.

Banerjee [7] applied the Timoshenko theory on a beam while coupling bending and torsion and considering rotational inertia. The homogenization theory is used to obtain the characteristics of the composite material.

Nilsson et al. [8] used equivalent homogeneous characteristics to study the bending vibrations of sandwich beams. The Hamilton's theory is applied, taking into account transverse shear, rotational inertia, and distortion. The dispersion results are given for three modes with different boundary conditions. Experimental results: natural 


\section{Nomenclature}

\begin{tabular}{|c|c|}
\hline$x, y, z$ & Cartesian coordinates \\
\hline$t$ & Time coordinate \\
\hline$W_{c}$ & Displacement of core in $z$ direction \\
\hline$W$ & Displacement of any point of the sandwich beam in $z$ direction \\
\hline$\varepsilon_{x B}, \varepsilon_{x B}^{\prime}$ & Bending strain in lower and upper skins, respectively \\
\hline$f_{l}, f_{u}$ & Thickness of lower and upper skins, respectively \\
\hline$E$ & Modulus of elasticity of skins \\
\hline$E_{c}$ & Modulus of elasticity of core \\
\hline$V_{\mathrm{BF}}, V_{\mathrm{BF}}^{\prime}$ & Energy of elastic bending of the lower and upper skins, respectively \\
\hline$a$ & Length of the sandwich beam \\
\hline$b$ & Width of the sandwich beam \\
\hline$c$ & Thickness of core \\
\hline$\nu$ & Poisson's ratio of skins \\
\hline$\sigma_{f}$ & Normal stress in an arbitrary fiber at $z$ coordinate \\
\hline$N_{\mathrm{FL}}, N_{F \mathrm{U}}$ & Normal force of the lower and upper skins, respectively \\
\hline$\varepsilon_{x L}, \varepsilon_{x U}$ & Strain in the $x$ direction of lower and upper skins, respectively \\
\hline$V_{\mathrm{CC}}$ & Shear deformation energy per width unit of core \\
\hline$V_{\mathrm{FC}}$ & Bending deformation energy per width unit of core \\
\hline$V_{\mathrm{NF}}, V_{\mathrm{NF}}^{\prime}$ & Tensile strain energy per width unit related to normal force in lower and upper skins, respectively \\
\hline$G_{x z}$ & Modulus of rigidity of core in $x z$ plane \\
\hline$\tau_{x z}$ & Shear stress in core \\
\hline$\gamma_{x z}$ & Shear strain in core \\
\hline$\rho$ & Mass density of the sandwich beam per width unit \\
\hline$\omega$ & Natural frequencies of the sandwich beam \\
\hline$V$ & Total elastic strain energy per width unit of beam \\
\hline$T$ & Kinetic energy per width unit of beam \\
\hline$E_{\mathrm{CT}}$ & Translational kinetic energy \\
\hline$E_{\mathrm{CR}}$ & Rotational kinetic energy \\
\hline$A_{1}, A_{2}, A_{3}, A_{4}, A_{5}, A_{6}$ & Constants \\
\hline
\end{tabular}

frequencies, modal shapes, and modal damping; are then presented to validate the theoretical results.

Blevins [9] presented a detailed list of formulas to determine the natural frequencies and modal shapes for various structures.

Fotsing et al. [10] studied experimentally the effect of adding viscoelatic layers on damping in sandwich beams.

Rayjade and Seshagiri Rao [11] presented the mechanical characteristics of a sandwich structure and analyzed the bending.

The paper studies the effect of displacement fields on the bending vibration of honeycomb sandwich beams. Indeed, several polynomial displacement fields are tested on two honeycomb sandwich beams: Nomex paper (soft material) and Aluminum alloy (hard material). The effect of core rotational inertia and bending energy on the sandwich beams vibration is also studied. The modal shapes obtained analytically are then compared to those obtained numerically. The experimental results allowed comparing the natural frequencies obtained analytically, numerically, and experimentally. Numerically obtained torsion results are also presented.

\section{Experimental study}

The experimental modal analysis is a reverse method which permits to derive from the responses of an excited structure, the structure characteristics.

The structure mounting method is very important. Most often beams are clamped-free (Cantilever). Depending on the configuration, modes are very different (especially the first one).

The tested honeycomb sandwich beams are clampedfree, their dimensions are $(\mathrm{mm}): a=250, b=53, c=$ 5 (Aluminum alloy, $f_{1, u}=0.75$ ) and 8 (Nomex paper, $\left.f_{1, u}=0.5\right)$.

\subsection{Test setup}

\subsubsection{Used equipment}

The test setup (Fig. 1) consists of a massive steel structure for embedding beams, isolated from external vibrations. The beam is mounted clamped-free, it is divided into six equal intervals to set the excitation and measurement points. The beam is excited on different points 


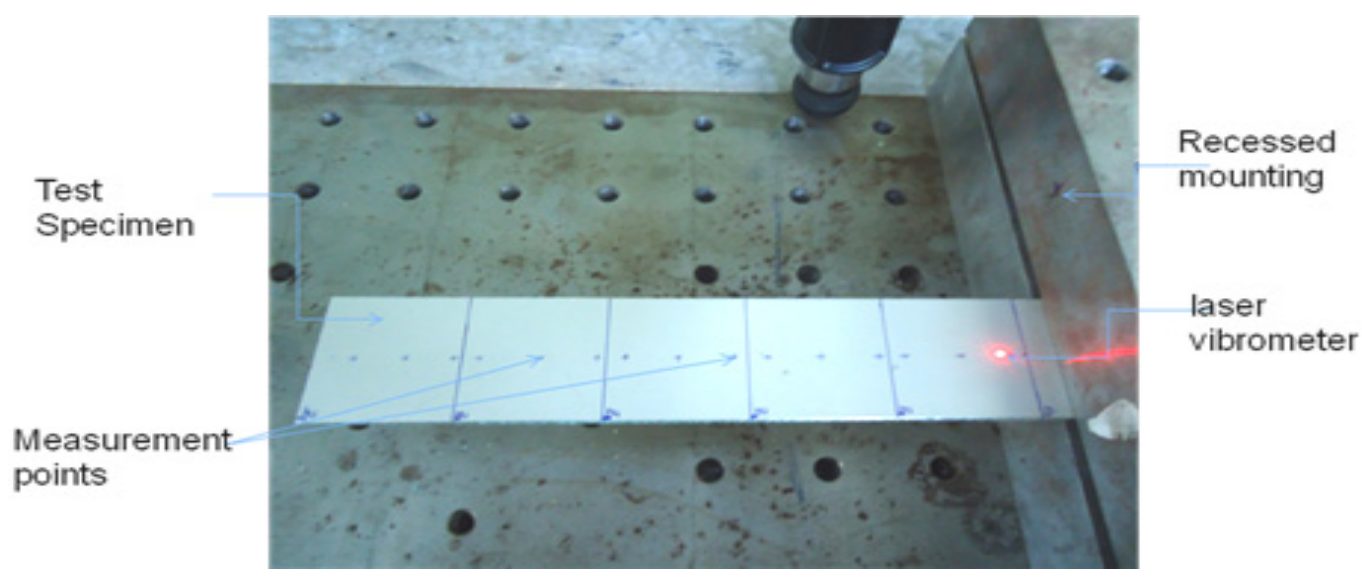

Fig. 1. Test setup photo.

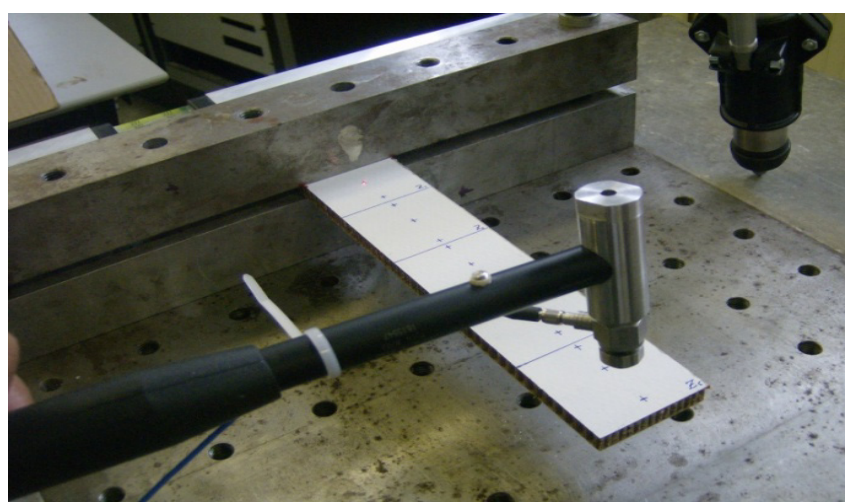

Fig. 2. Excitation with shock sensor hammer.

using an instrumented hammer connected to an acquisition system. The vibrational response of the beam is then measured on different points using a laser vibrometer.

Performing a test of static bending using gradually increasing shear force (the specimen is relatively thick) allows determining the static stiffness $E_{I z}$ (Beam bending stiffness). The specimen is assumed to be homogeneous, isotropic, elastic, and linear.

\subsubsection{Testing procedure}

Modal analysis is carried out for the sandwich beam bending vibration. The frequency response to a pulse, produced using the shock hammer (Fig. 2), allows determining the first natural frequency of the tested beam.

The experimental modal analysis with impact excitation presents the advantage of being fairly simple to implement. Figure 3 explains the experimental setup used to study the bending vibration of the cantilever honeycomb sandwich beams. The Beam is excited at a point using a shock hammer (BKV 06) and the response is detected at a different point using a laser vibrometer (PDV 100). The vibrometer consists of an optical head associated with a controller. The shock sensor and the laser vibrometer signals are then digitized and processed by a dynamic analyzer (OROS).

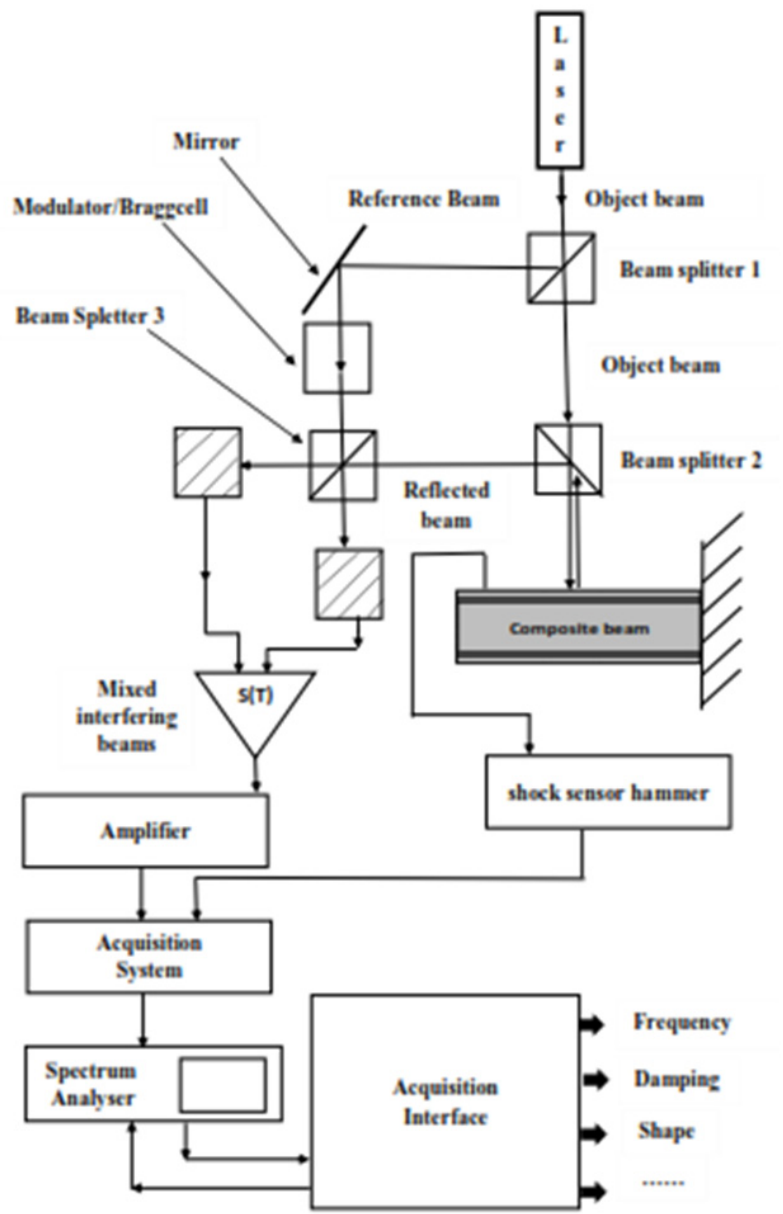

Fig. 3. Test setup diagram.

The analyzer comprises an acquisition and processing board, equipped with control and signal processing software. The acquisition board processor enables simultaneous acquisition of two signals. The system performs signal acquisition, controls vesting conditions (sensitivity, bandwidth, trigger conditions, etc.), and performs signal processing (Fourier transform, frequency response, etc.). 

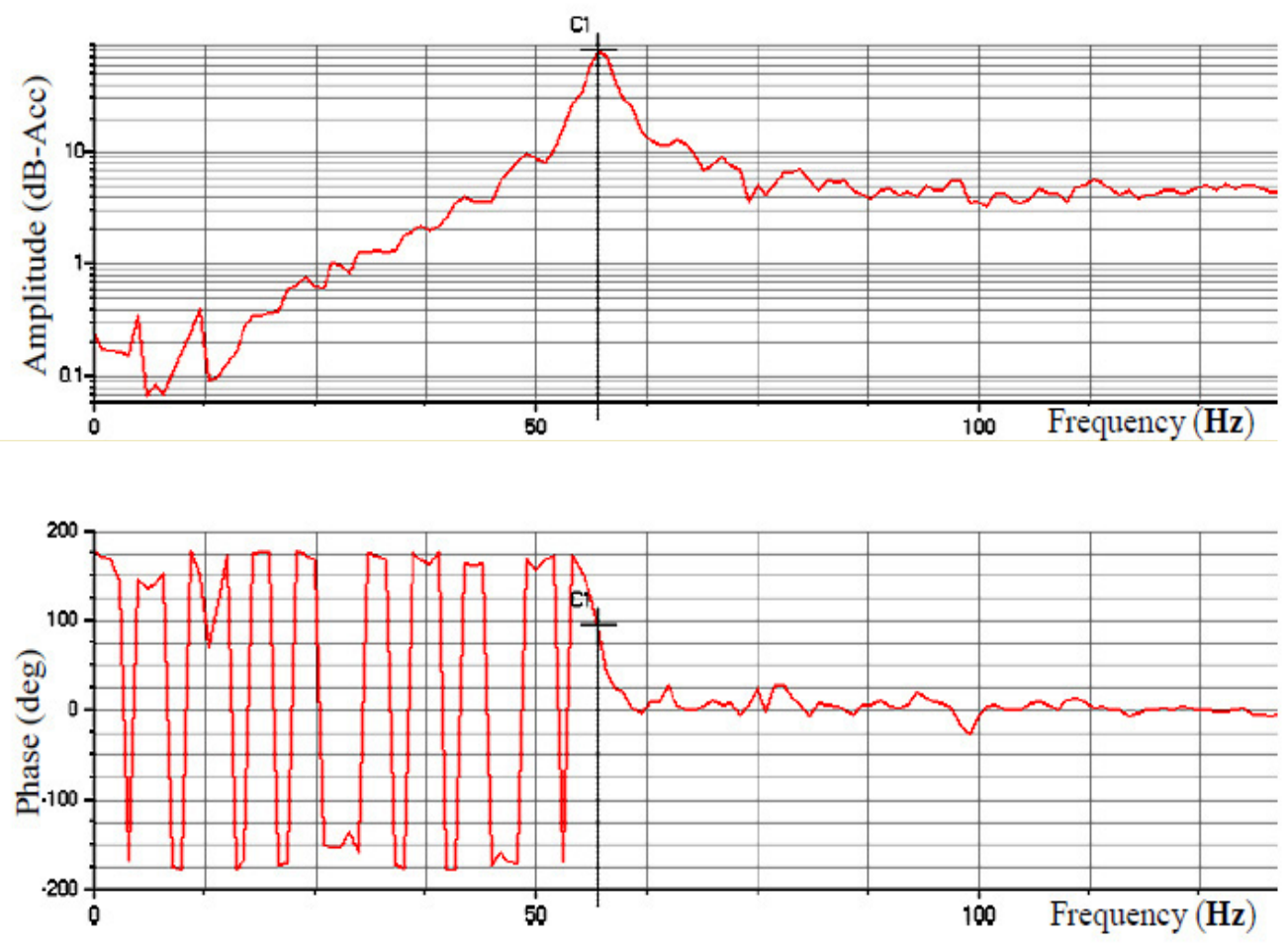

Fig. 4. Transfer function (Amplitude and Phase) of the Aluminum bea.

The signals and associated treatments can be saved for post-processing.

The excitation hammer with rubber tip and incorporated force sensor excites the beam vertically (the beam is mounted horizontally) with a transient load of small amplitude to conserve the linearity. The bi-channel spectrum analyzer calculates the average transfer function over several tests. The coherence function is checked at each test.

\subsection{Test results}

The case where the excitation is at the free end $(x=a)$ and the measurement is close to the clamped end $(x=$ $a / 6)$ is retained ( $a$ : The beam length).

The obtained transfer functions, in amplitude and phase, of the tested sandwich beams are shown in Figures 4 and 5 (Frequency band: 0-256 Hz; Sampling: $0.5 \mathrm{~Hz}$; Windows: Hamming).

The extraction of the modal parameters from the measurements is based on the representation of the behavior from modal impulse responses. They are obtained by multi-excitation and the impulse responses are calculated by inverse Fourier transform of the transfer functions. Sometimes the analysis of the transfer functions is carried out by adjusting the experimental curves using the least square method (to smoothen the transfer functions).

The peak amplitude method is used for the first modes (lowly damped), the obtained natural frequencies are (Root Mean Square, over eight results): $f 1=57 \mathrm{~Hz}$ (Aluminum beam), $f 1=23 \mathrm{~Hz}$ (Nomex beam).

\section{Numerical study}

Using the finite element code Abaqus, the eigen value problem was obtained by a finite element discretization using a three-dimensional, 8-node, linear, and isoparametric element (hexahedral) with reduced integration (C3D8R). Each node has three translational degrees of freedom. The Hourglass phenomena must not be a problem because of the important number of elements.

$$
\left\{[K]-\omega^{2}[M]\right\}\{Y\}=0
$$

Where $M$ is the beam mass matrix, $K$ is the beam stiffness matrix, and $\omega$ is the angular frequency. For this study, the problem (1) was symmetrized, the eigenvector $\{U\}$ can be determined using two approaches: Lanczos method or subspace iteration. The Lanczos method was adopted, and the solutions will be discussed.

The modeling and simulation are made following these steps:

- Geometric modeling of the sandwich beam under the module Part (core and skins).

- Materials creation of the sandwich beam under the module Property (the beam mechanical characteristics are shown in Tab. 1).

- Assembling of the sandwich beam under the module Assembly (the option Depending is selected to facilitate the meshing of each element apart).

- Definition of step analysis of the sandwich beam under the module Steps. 


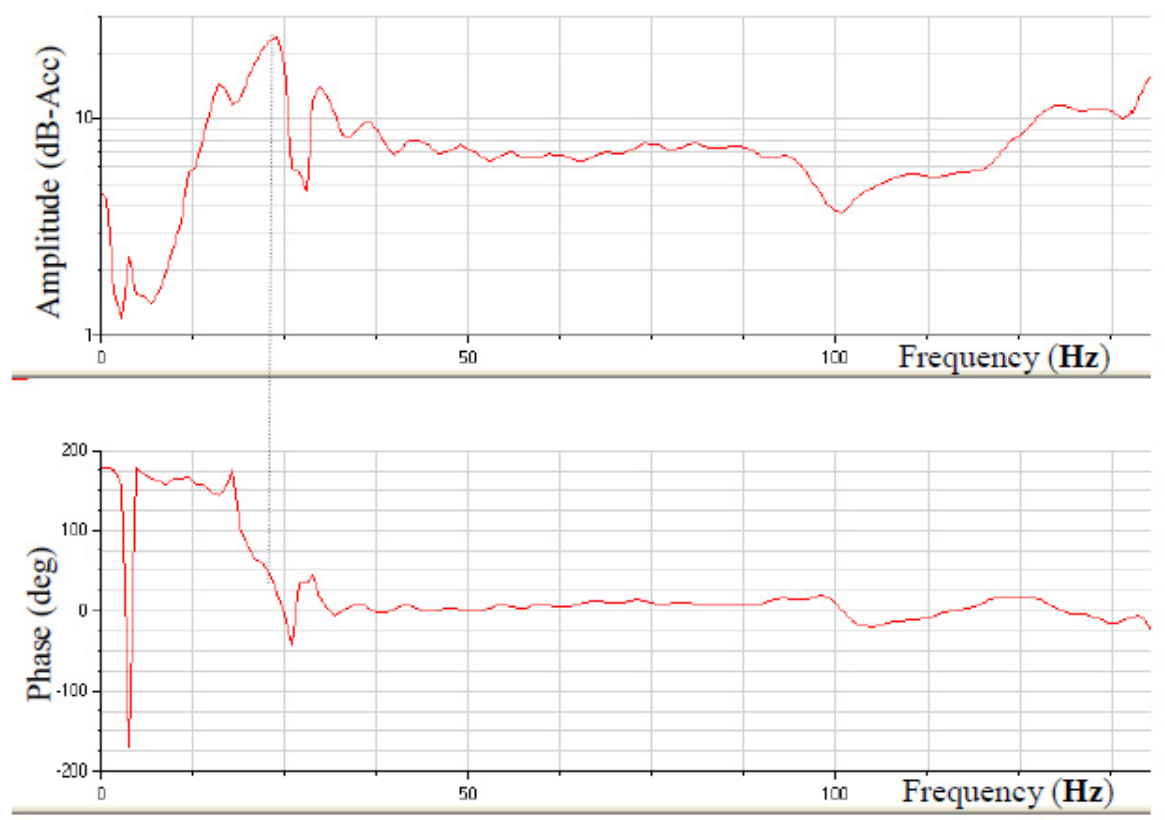

Fig. 5. Transfer function (Amplitude and Phase) of the Nomex beam.

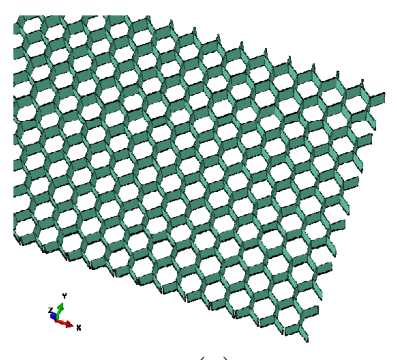

(a)

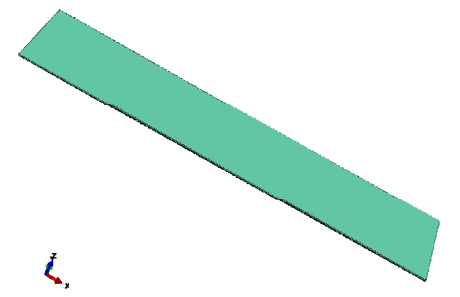

(b)

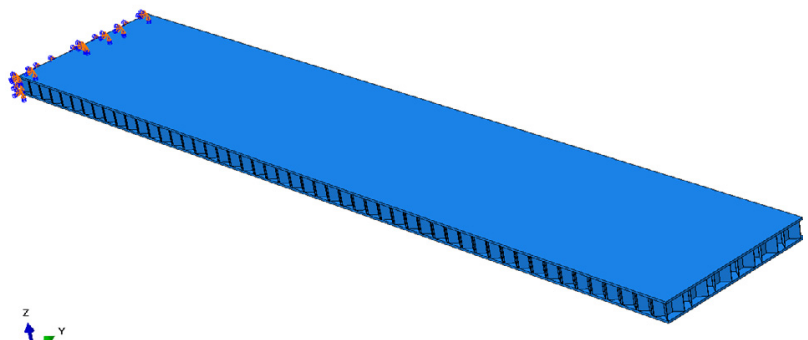

Fig. 8. Mounted beam.

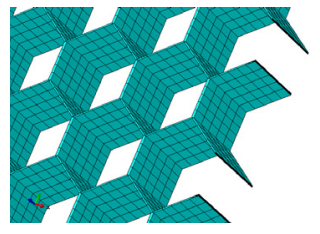

(a)

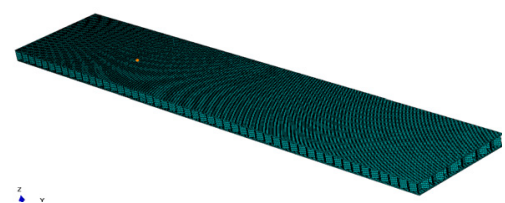

(b)
Fig. 7. Assembled beam.

- Application of the boundary conditions under the module Load.

Fig. 9. Meshed core (a) and beam (b).

\subsection{Bending}

\subsubsection{Bending in Plane $(x, z)$}

Figures 10-14. wich beam is meshed choosing the meshing technique: Structured. Hexahedral C3D8R element is used. The chosen approximate mesh size is around 0.05 (Seed/Part), then the meshing is run on the whole beam).

- Analytical work creation and submission under the module Jobs.

- Results presentation in the module Visualization (Aluminum beam). 

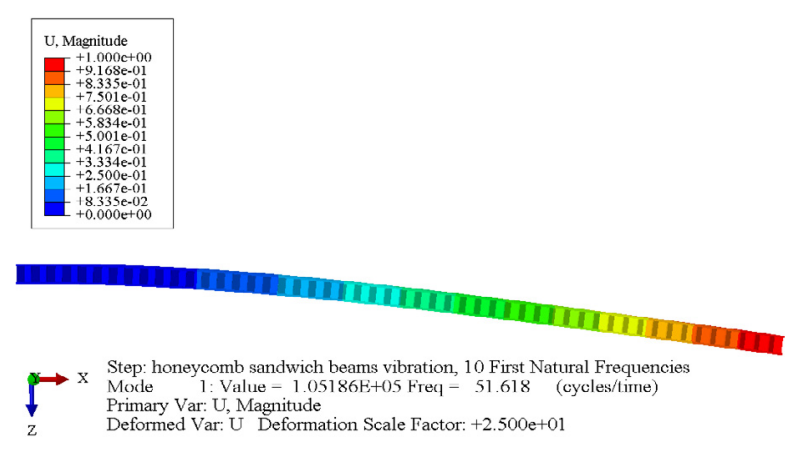

Fig. 10. First mode shape of bending in plane $(x, z)$.

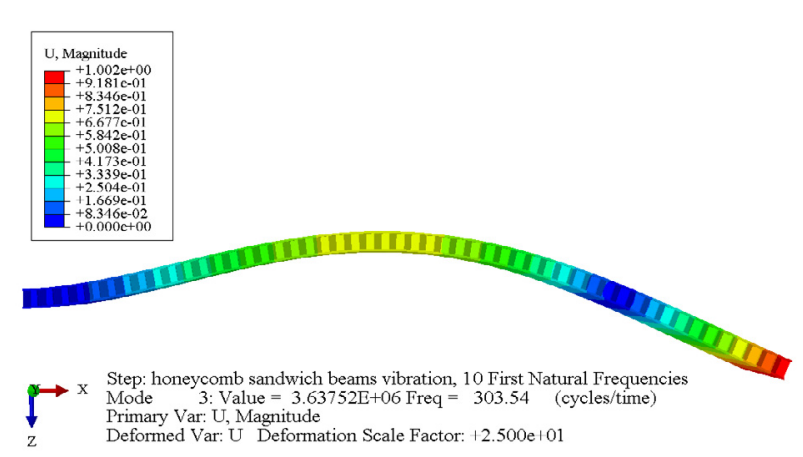

Fig. 11. Second mode shape of bending in plane $(x, z)$.

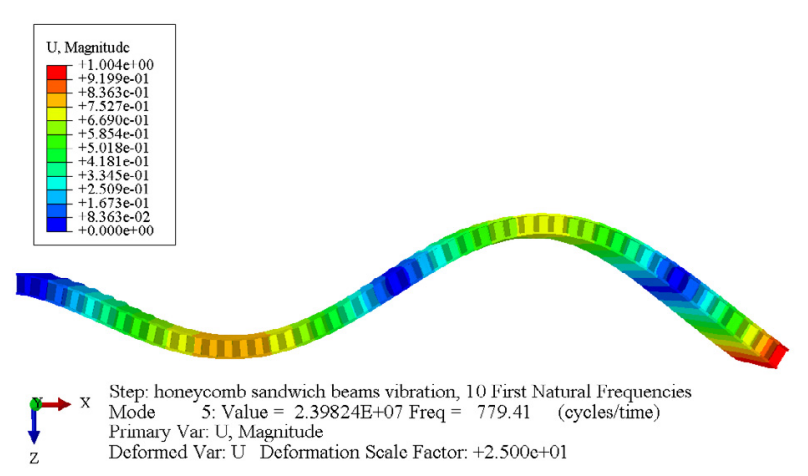

Fig. 12. Third mode shape of bending in plane $(x, z)$.

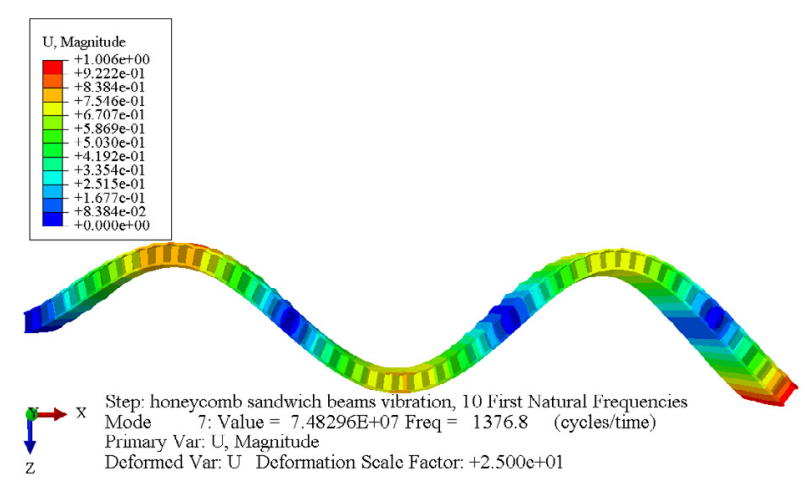

Fig. 13. Fourth mode shape of bending in plane $(x, z)$.

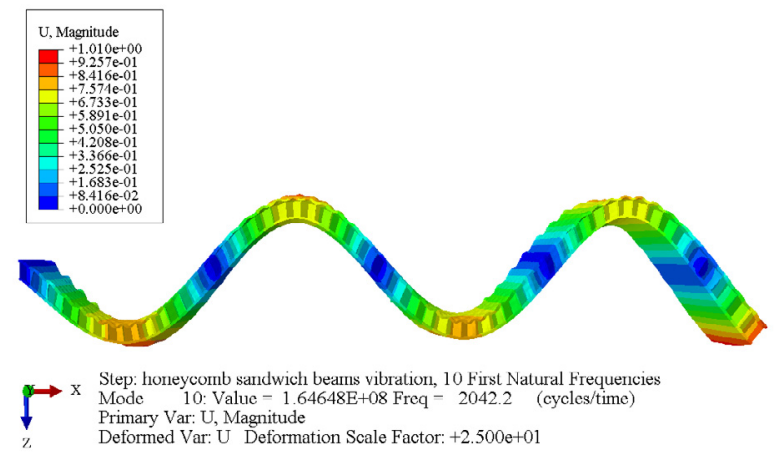

Fig. 14. Fifth mode shape of bending in plane $(x, z)$.

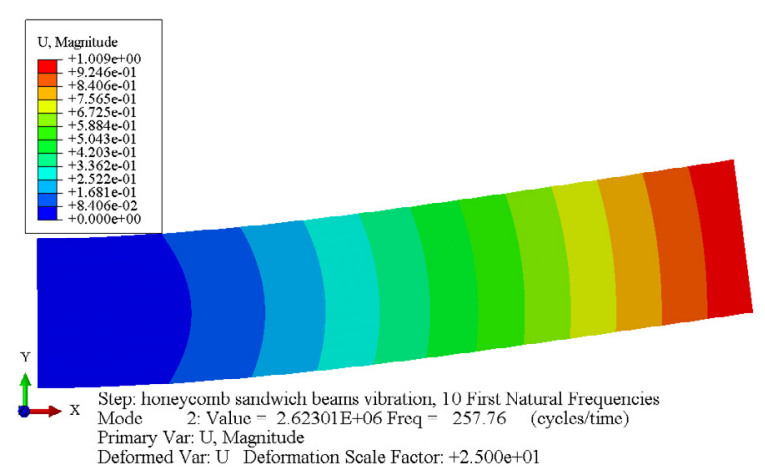

Fig. 15. First mode shape of bending in plane $(x, y)$.

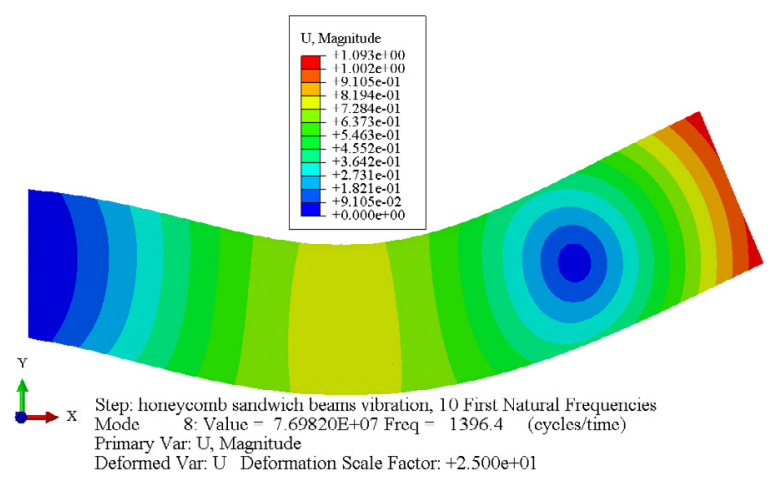

Fig. 16. Second mode shape of bending in plane $(x, y)$.

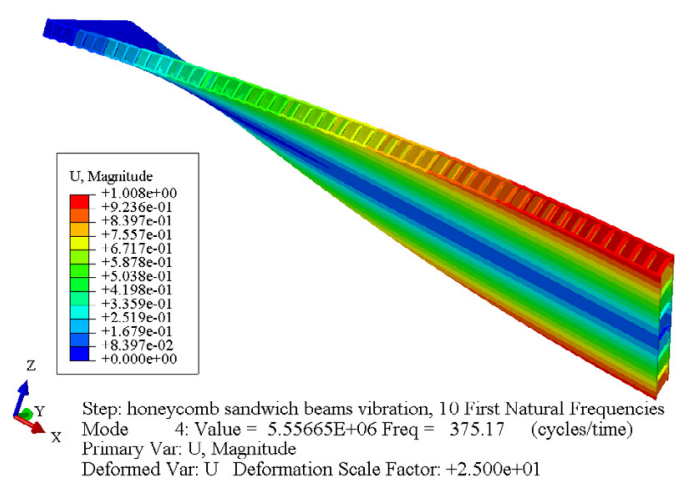

Fig. 17. First mode shape of torsion. 


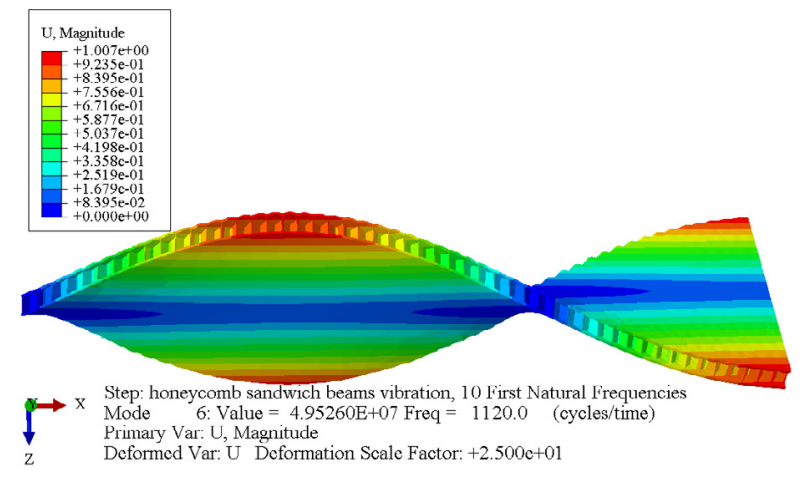

Fig. 18. Second mode shape of torsion.

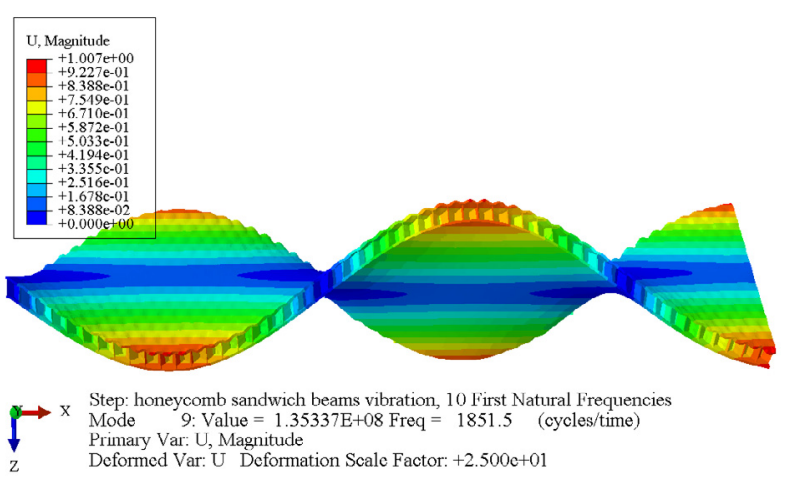

Fig. 19. Third mode shape of torsion.

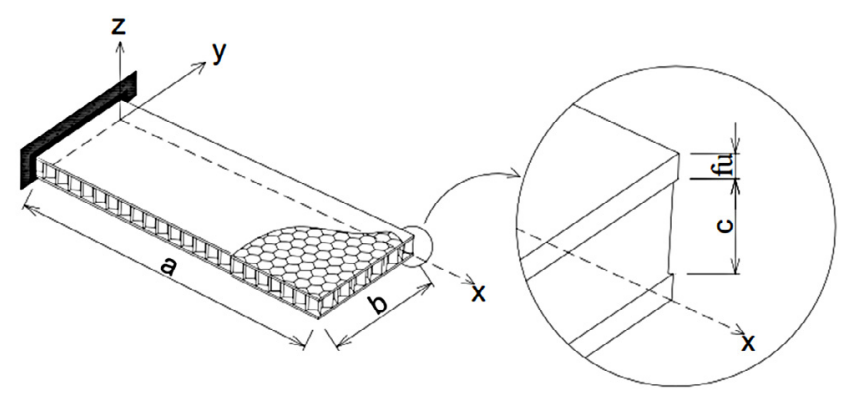

Fig. 20. Honeycomb sandwich beam.

\section{Analytical study}

\subsection{Mathematical formulation}

The studied honeycomb sandwich beam is composed of two skins made of a material having good mechanical properties, and a core made of a material having low mechanical properties but shaped in honeycomb. The three components are perfectly bonded to make a material having very good mechanical properties.

The displacement fields are chosen respecting the boundary conditions.

The potential energy of the sandwich beam is the sum of the bending and tensile elastic deformation energy of both upper and lower skins, as well as the shear and bending energy of core.

The bending elastic deformation $\left(\varepsilon_{x B}, \varepsilon_{x B}^{\prime}\right)$ and energy $\left(V_{\mathrm{BF}}, V_{\mathrm{BF}}^{\prime}\right)$ for each of the lower and upper skins are [12]:

$$
\varepsilon_{x B}=\left(z+\frac{f_{l}}{2}\right)\left(\frac{\partial^{2} W_{c}}{\partial x^{2}}\right)
$$

and

$$
\begin{gathered}
\varepsilon_{x B}^{\prime}=\left(z-c-\frac{f_{u}}{2}\right)\left(\frac{\partial^{2} W_{c}}{\partial x^{2}}\right) \\
V_{\mathrm{BF}}=\frac{E}{2\left(1-\nu^{2}\right)} \int_{f_{l}}^{0} \mathrm{~d} z \int_{0}^{a}\left(\varepsilon_{x B}\right)^{2} \mathrm{~d} x
\end{gathered}
$$

and

$$
V_{\mathrm{BF}}^{\prime}=\frac{E}{2\left(1-\nu^{2}\right)} \int_{c}^{c+f_{u}} \mathrm{~d} z \int_{0}^{a}\left(\varepsilon_{x B}^{\prime}\right)^{2} \mathrm{~d} x
$$

To determine the tensile energy of each skin, we must first determine the normal force applied to its external surface and its extension in the $x$ direction. The normal Forces $\left(N_{\mathrm{FL}}, N_{\mathrm{FU}}\right)$ at each of the lower and upper skins are:

$$
N_{\mathrm{FL}}=\int_{f_{l}}^{0} \sigma_{f} \mathrm{~d} z
$$

and

$$
N_{\mathrm{FU}}=\int_{c}^{c+f_{u}} \sigma_{f} \mathrm{~d} z
$$

The expression of the stress in an arbitrary point of the skin $\left(\sigma_{f}\right)$ is:

$$
\sigma_{f}=-E\left(z-\frac{c}{2}\right)\left(\frac{\partial^{2} W_{c}}{\partial x^{2}}\right)
$$

Substituting (7) in (5) and (6), the normal forces become:

$$
N_{\mathrm{FL}}=-\int_{f_{l}}^{0} E\left(z-\frac{c}{2}\right)\left(\frac{\partial^{2} W_{c}}{\partial x^{2}}\right) \mathrm{d} z
$$

and

$$
N_{\mathrm{FU}}=-\int_{c}^{c+f_{u}} E\left(z-\frac{c}{2}\right)\left(\frac{\partial^{2} W_{c}}{\partial x^{2}}\right) \mathrm{d} z
$$

The normal forces of the upper and lower skins after integration, are:

$$
N_{\mathrm{FL}}=E \frac{f_{l}}{2}\left(c+f_{l}\right)\left(\frac{\partial^{2} W_{c}}{\partial x^{2}}\right)
$$

and

$$
N_{\mathrm{FU}}=-E \frac{f_{u}}{2}\left(c+f_{u}\right)\left(\frac{\partial^{2} W_{c}}{\partial x^{2}}\right)
$$

The tensile energies $\left(V_{N F}, V_{N F}^{\prime}\right)$ of each of the lower and upper skins related to the normal forces are:

$$
V_{\mathrm{NF}}=\frac{1}{2} \int_{0}^{a} N_{\mathrm{FL}} \varepsilon_{x L} \mathrm{~d} x
$$


and

$$
V_{\mathrm{NF}}^{\prime}=\frac{1}{2} \int_{0}^{a}\left(-N_{\mathrm{FL}}\right) \cdot\left(-\varepsilon_{x L}\right) \mathrm{d} x
$$

where,

$\varepsilon_{x L}$ : Elastic deformation in the $x$ direction of the lower skin.

$\varepsilon_{x U}$ : Elastic deformation in the $x$ direction of the upper skin.

The elastic bending deformations of each of the lower and upper skins are:

$$
\varepsilon_{x L}=\frac{1}{2}\left(c+f_{l}\right)\left(\frac{\partial^{2} W_{c}}{\partial x^{2}}\right)
$$

and

$$
\varepsilon_{x U}=-\frac{1}{2}\left(c+f_{u}\right)\left(\frac{\partial^{2} W_{c}}{\partial x^{2}}\right)
$$

Substituting (10) and (14) in (12), we obtain:

$$
V_{\mathrm{NF}}=\frac{E \cdot f_{l} \cdot\left(c+f_{l}\right)^{2}}{8} \int_{0}^{a}\left(\frac{\partial^{2} W_{c}}{\partial x^{2}}\right) \mathrm{d} x
$$

where, Substituting (11) and (15) in (13), we obtain:

$$
V_{\mathrm{NF}}^{\prime}=\frac{E \cdot f_{u} \cdot\left(c+f_{u}\right)^{2}}{8} \int_{0}^{a}\left(\frac{\partial^{2} W_{c}}{\partial x^{2}}\right) \mathrm{d} x
$$

The core shear energy $\left(V_{c c}\right)$ is:

$$
V_{c}=\frac{1}{2} \int_{0}^{c} \mathrm{~d} z \int_{0}^{a} G_{X Z} \cdot\left(\gamma_{X Z}\right)^{2} \mathrm{~d} x
$$

where,

$$
\gamma_{X Z}=\frac{1}{G_{X Z}} \cdot \tau_{X Z}
$$

and

$$
\tau_{X Z} \mathrm{~d} x=-\frac{\partial}{\partial x} N_{\mathrm{FL}} \mathrm{d} x
$$

The core bending energy $\left(V_{\mathrm{FC}}\right)$ is:

$$
V_{\mathrm{FC}}=\frac{1}{2} \int_{0}^{a} E_{c} \cdot I \cdot\left(\frac{\partial^{2} W_{c}}{\partial x^{2}}\right) \mathrm{d} x \text { with } I=\frac{c^{3} \cdot b}{12}
$$

The total potential energy is therefore:

$$
V=V_{\mathrm{CC}}+V_{\mathrm{FC}}+V_{\mathrm{NF}}+V_{\mathrm{NF}}^{\prime}+V_{\mathrm{BF}}+V_{\mathrm{BF}}^{\prime}
$$

The total kinetic energy of the sandwich beam is equal to the sum of translational kinetic energy and rotational kinetic energy $\left(E_{\mathrm{CT}}, E_{\mathrm{CR}}\right)$ :

$$
T=E_{\mathrm{CT}}+E_{\mathrm{CR}}
$$

where

$$
E_{\mathrm{CT}}=\frac{1}{2} \rho \int_{0}^{a}\left(\frac{\partial^{2} W_{c}}{\partial x^{2}}\right) \mathrm{d} x
$$

and

$$
E_{\mathrm{CR}}=\frac{1}{2} \int_{0}^{a} I\left(\frac{\partial}{\partial t}\left(\frac{\partial W}{\partial x}\right)\right)^{2} \mathrm{~d} x
$$

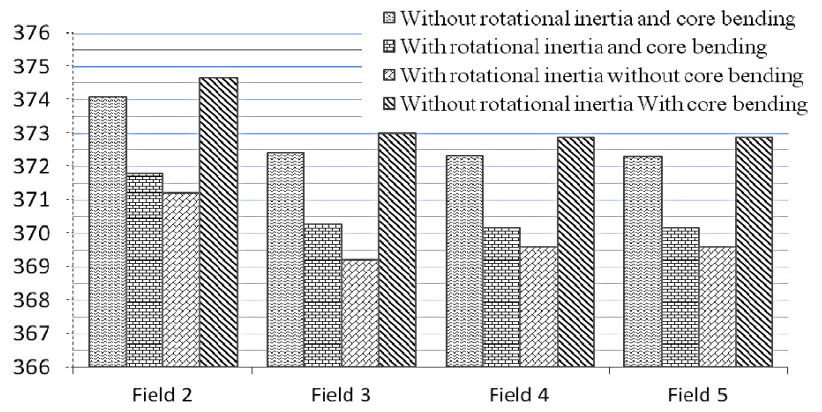

Fig. 21. First natural angular frequency $(\mathrm{rad} / \mathrm{s})$ function of the considered parameters.

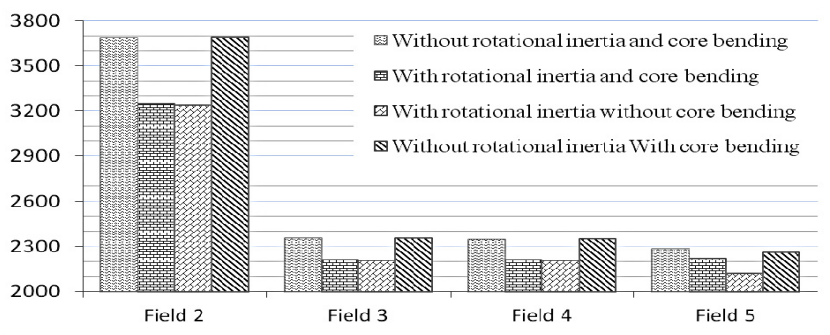

Fig. 22. Second natural angular frequency $(\mathrm{rad} / \mathrm{s})$ function of the considered parameters.

The beam is considered as a conservative system, hence $(i=1 \ldots n-1)$ are constants. Resolving the obtained system allows to determine the first $(n-1)$ natural frequencies and their modal shapes.

The resolution for each of the displacement fields gives a Cramer system of equations. An example of Crammer systems is presented hereafter:

$$
\text { See equation next page. }
$$

where: $\quad X 21, X 22, X 23, X 24, X 25, Y 21, Y 22, Y 23, Y 24$, $Y 25, Z 21, Z 22, Z 23, Z 24, Z 25, U 21, U 22, U 23, U 24, U 25$, $V 21, V 22, V 23, V 24, V 25$ and $k_{\mathrm{ij}}$ are listed in the Appendix.

\subsection{Analytical results and comparison}

The mechanical and geometrical characteristics of the tested beams, are as follows (Tab. 1).

The considered displacement fields are as follows:

Field 1: $W=W_{c}=\left(A_{1} x^{2}\right) \sin (\omega t)$

Field 2: $W=W_{c}=\left(A_{1} x^{2}+A_{2} x^{3}\right) \sin (\omega t)$

Field 3: $W=W_{c}=\left(A_{1} x^{2}+A_{2} x^{3}+A_{3} x^{4}\right) \sin (\omega t)$

Field 4: $W=W_{c}=\left(A_{1} x^{2}+A_{2} x^{3}+A_{3} x^{4}+A_{4} x^{5}\right) \sin (\omega t)$

Field 5: $W=W_{c}=\left(A_{1} x^{2}+A_{2} x^{3}+A_{3} x^{4}+A_{4} x^{5}+\right.$ $\left.A_{5} x^{6}\right) \sin (\omega t)$

\section{Core rotational inertia and bending (Aluminum beam)}

Figures 21 and 22 illustrate the first and second natural frequencies for different order of displacement fields and with or without rotational inertia and core bending. 
I. Chenini et al.: Mechanics \& Industry 18, 302 (2017)

$$
\left[\begin{array}{ccccc}
X 21-\frac{1}{2} \rho \omega^{2} \cdot k_{11} & X 22-\frac{1}{2} \rho \omega^{2} \cdot k_{12} & X 23-\frac{1}{2} \rho \omega^{2} \cdot k_{13} & X 24-\frac{1}{2} \rho \omega^{2} \cdot k_{14} & X 25-\frac{1}{2} \rho \omega^{2} \cdot k_{15} \\
Y 21-\frac{1}{2} \rho \omega^{2} \cdot k_{21} & Y 22-\frac{1}{2} \rho \omega^{2} \cdot k_{22} & Y 23-\frac{1}{2} \rho \omega^{2} \cdot k_{23} & Y 24-\frac{1}{2} \rho \omega^{2} \cdot k_{24} & Y 25-\frac{1}{2} \rho \omega^{2} \cdot k_{25} \\
Z 21-\frac{1}{2} \rho \omega^{2} \cdot k_{31} & Z 22-\frac{1}{2} \rho \omega^{2} \cdot k_{32} & Z 23-\frac{1}{2} \rho \omega^{2} \cdot k_{33} & Z 24-\frac{1}{2} \rho \omega^{2} \cdot k_{34} & Z 25-\frac{1}{2} \rho \omega^{2} \cdot k_{35} \\
U 21-\frac{1}{2} \rho \omega^{2} \cdot k_{41} & U 22-\frac{1}{2} \rho \omega^{2} \cdot k_{42} & U 23-\frac{1}{2} \rho \omega^{2} \cdot k_{43} & U 24-\frac{1}{2} \rho \omega^{2} \cdot k_{44} & U 25-\frac{1}{2} \rho \omega^{2} \cdot k_{45} \\
V 21-\frac{1}{2} \rho \omega^{2} \cdot k_{51} & V 22-\frac{1}{2} \rho \omega^{2} \cdot k_{52} & V 23-\frac{1}{2} \rho \omega^{2} \cdot k_{53} & V 24-\frac{1}{2} \rho \omega^{2} \cdot k_{54} & V 25-\frac{1}{2} \rho \omega^{2} \cdot k_{55}
\end{array}\right]\left\{\begin{array}{l}
A_{1} \\
A_{2} \\
A_{3} \\
A_{4} \\
A_{5}
\end{array}\right\}=\left\{\begin{array}{l}
0 \\
0 \\
0 \\
0 \\
0
\end{array}\right\}
$$

Table 1. Geometrical and mechanical characteristics of the tested beams.

\begin{tabular}{ccc}
\hline & Aluminum beam & NOMEX beam \\
\hline Length (a) in mm & 250 & \\
Width (b) in mm & 53 & 53 \\
Thickness of core (c) in mm & 5 & 8 \\
Thickness of skins in mm & 0.75 & 0.5 \\
Density $(\rho)$ in $\mathrm{Kg} / \mathrm{m}^{3}$ & 573 & 221 \\
$E_{c}$ : Elastic modulus of core in $\mathrm{MPa}$ & 130 & 2.5 \\
$E:$ Elastic modulus of skins in MPa & 70.103 & 8.63 \\
$G_{\mathrm{XZ}}$ : Shear modulus of core in MPa & 5600 & $70 \times 10^{3}$ \\
$\nu$ : Poisson's ratio & 0.33 & 0.33 \\
\hline
\end{tabular}
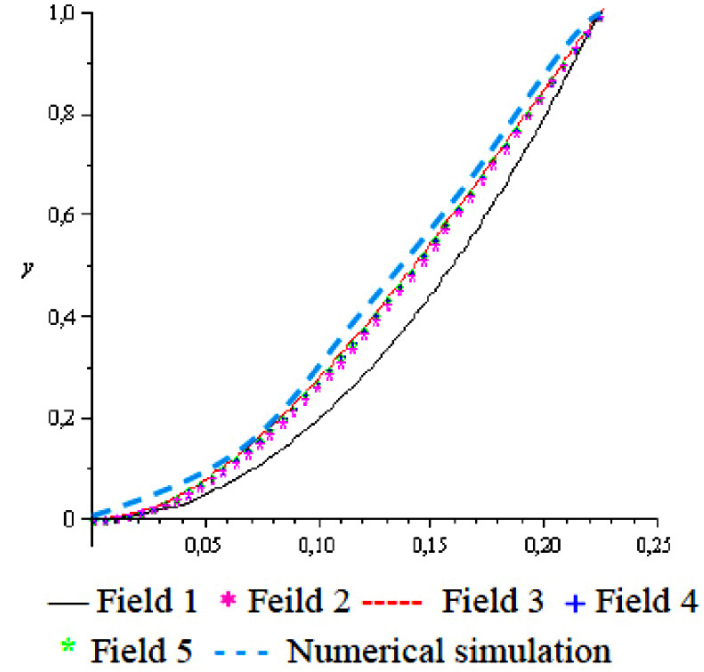

Fig. 23. First mode shape for different fields.

It is notable that the first natural frequency obtained with the first displacement field is widely superior to the others, and tends to the value of $372(\mathrm{rad} / \mathrm{s})$ considering both rotational inertia and core bending, from the third field. Also, the second natural frequency tends to the value of $2360(\mathrm{rad} / \mathrm{s})$ considering rotational inertia and core bending, from the third field to the fourth field.

It can be concluded that the accuracy of the natural frequency depends on the order of the displacement field, but also on the core rotational inertia and bending.

\section{Modal shapes (Aluminum beam)}

Figures 23-26 illustrate the first four modal shapes for the sandwich beam using the same scale for all the displacement fields and including the numerical simulation.

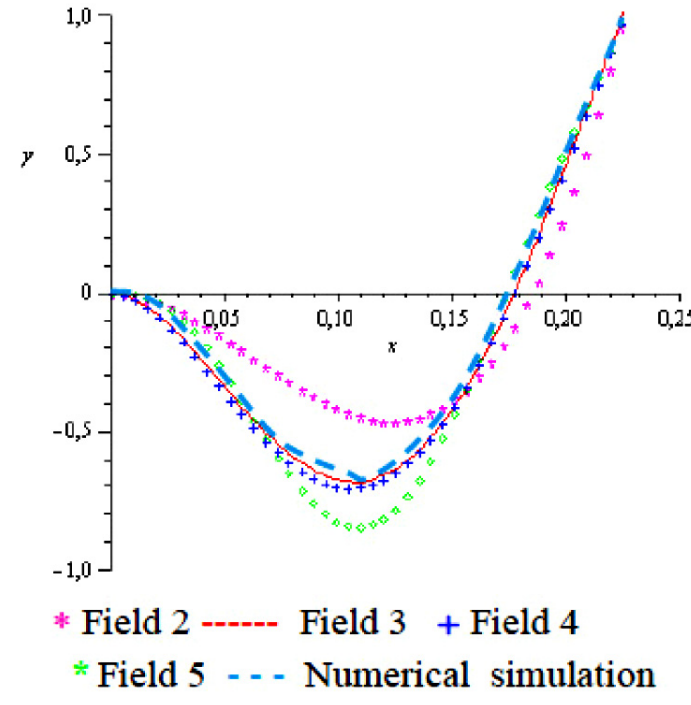

Fig. 24. Second mode shape for different fields.

It is notable that when the mode order increases, the difference between all the results is more significant. However, the first analytical two modal shapes are close to the numerical simulation when using an adequate displacement field.

It can be concluded that the results for modal shapes are close to those obtained for natural frequencies. The displacement field order is to be considered cautiously.

\section{Natural frequencies}

Table 2 presents the obtained natural frequencies in bending and in torsion of the two sandwich beams.

It is noted that all the results are close for the first four frequencies but not for the fifth frequency. This means that the displacement field considered for the fifth fre- 
I. Chenini et al.: Mechanics \& Industry 18, 302 (2017)

Table 2. Comparison of the frequencies for the three used methods.

\begin{tabular}{|c|c|c|c|c|c|c|c|c|}
\hline & \multicolumn{2}{|c|}{ Experimental } & \multicolumn{2}{|c|}{ Numerical } & \multicolumn{2}{|c|}{ Analytical } & & \\
\hline & $\mathrm{Al}$ & Nomex & $\mathrm{Al}$ & Nomex & $\mathrm{Al}$ & Nomex & & \\
\hline \multirow{7}{*}{ Bending in Plane } & \multirow{5}{*}{$(x, z)$} & f1 & 57 & 23 & 52 & 26 & 58 & 32 \\
\hline & & $\mathrm{f} 2$ & & & 304 & 187 & 337 & 199 \\
\hline & & f3 & & & 779 & 365 & 795 & 488 \\
\hline & & $\mathrm{f} 4$ & & & 1372 & 565 & 1268 & 664 \\
\hline & & $\mathrm{f} 5$ & & & 2042 & 810 & 4015 & \\
\hline & \multirow{2}{*}{$(x, y)$} & f1 & & & 258 & 123 & 285 & \\
\hline & & f2 & & & 1396 & 572 & 1323 & \\
\hline \multirow{3}{*}{ Torsion } & & f1 & & & 375 & 165 & & \\
\hline & $\mathrm{f} 2$ & & & 1120 & 516 & & & \\
\hline & f3 & & & 1852 & 721 & & & \\
\hline
\end{tabular}

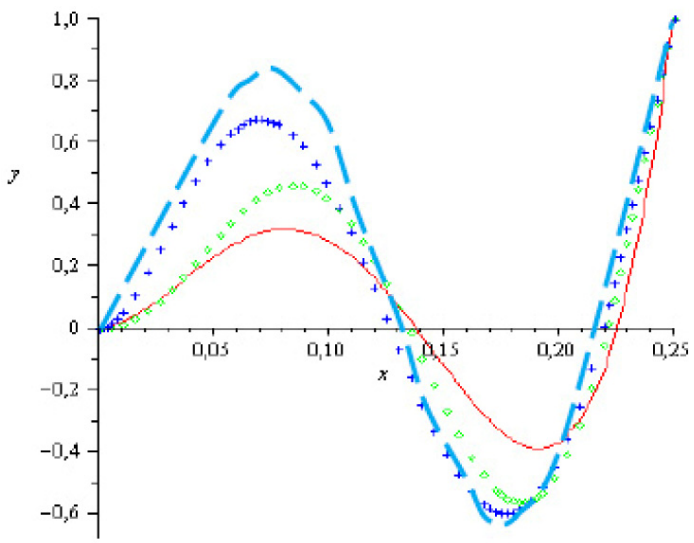

Field $3+$ Field $4 \div$ Field 5

- - - Numerical simulation

Fig. 25. Third mode shape for different fields.

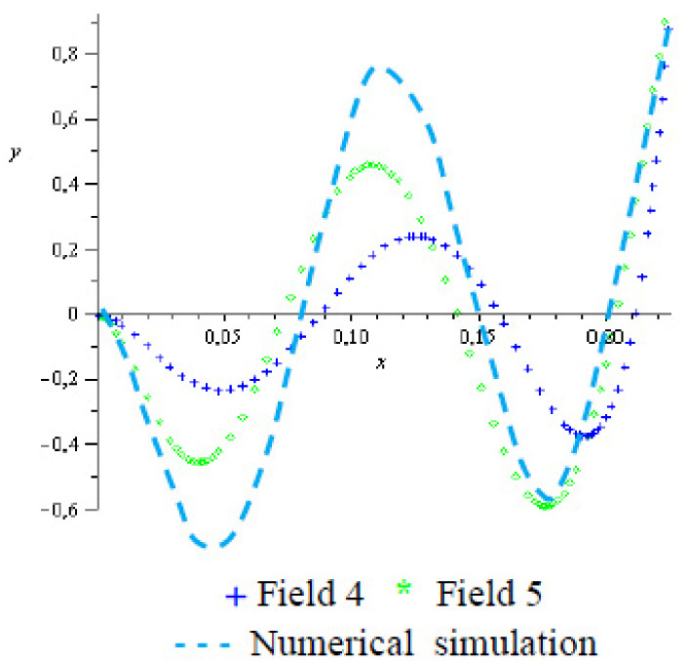

Fig. 26. Fourth mode shape for different fields.

quency is inadequate to represent accurately the beam vibration, thus a higher order displacement field is necessary.

It is also noted that the relative error between analytical and numerical results is around $10 \%$. The analytical results are higher than simulation results for the first four frequencies, it is necessary to introduce other parameters like modal mass and modal stiffness to explain the difference.

\section{Future works}

For each form of the displacement fields, we note a relationship between the coefficients of the mass matrix, which may be written as follows.

For $n$ order polynomial: $M_{\mathrm{ij}}=K_{\mathrm{ij}}-L_{\mathrm{ij}}$, with $i=$ $1 \ldots(n-1), j=1 \ldots(n-1)$, where $L_{\mathrm{ij}}$ can be in a general form: $L_{\mathrm{ij}}=\frac{1}{2} \rho \omega^{2} \frac{2}{(3+i+j)} a^{3+i+j)}$.

And: $K_{\mathrm{ij}}=4(1+j) a^{j} . \mathrm{C} ; K_{2 j}=N_{2 j}+(12 \cdot j) \cdot a^{j+1} . \mathrm{C}$.

The relations between thecoefficients are to be defined.

We can also choose a field of displacement according to the three directions $(x, y, z)$ for better description of the vibration of the sandwich beam, taking into account the torsion and the bending in both horizontal and vertical planes.

We can also introduce the structural damping similarly. The glue viscoelasticity can also be considered.

\section{Conclusion}

The paper studied the bending vibration of honeycomb sandwich beams and the effect of displacement fields. Indeed, it presented experimental, numerical, and analytical results of bending vibration of two honeycomb beams: Nomex paper and Aluminum alloy. The comparison of natural frequencies and modal shapes shows the kinematics effect on the sandwich beam vibration.

Comparing the obtained results, a slight difference is noted between the natural frequencies and modal shapes obtained numerically, analytically, and experimentally. The structural damping, the glue viscoelasticity, and the clamping imperfection although the taken measures, may be among the sources of the difference between theoretical and experimental results. Besides, the Nomex paper beam (soft material) conclusions are similar to those noted for the Aluminum beam (hard material).

The displacement field has notable influence on the natural frequencies and modal shapes, a righteous choice is determinant to describe accurately the vibrational behavior of the sandwich beam. 


\section{Appendix}

$$
\begin{aligned}
& X 21=\frac{8 a E\left(f_{l}\right)^{3}}{24\left(1-\nu^{2}\right)}+\frac{8 a E\left(f_{u}\right)^{3}}{24\left(1-\nu^{2}\right)}+\frac{8 a E \cdot f_{l}\left(f_{l}+c\right)^{2}}{8}+\frac{8 a E \cdot f_{u}\left(f_{u}+c\right)^{2}}{8}+\frac{8 a \cdot E_{C} \cdot c^{3}}{12} \\
& X 22=\frac{12 a^{2} E\left(f_{l}\right)^{3}}{24\left(1-\nu^{2}\right)}+\frac{12 a^{2} E\left(f_{u}\right)^{3}}{24\left(1-\nu^{2}\right)}+\frac{12 a^{2} E \cdot f_{l}\left(f_{l}+c\right)^{2}}{8}+\frac{12 a^{2} E \cdot f_{u}\left(f_{u}+c\right)^{2}}{8}+\frac{12 a^{2} \cdot E_{C} \cdot c^{3}}{12} \\
& X 23=\frac{16 a^{3} E\left(f_{l}\right)^{3}}{24\left(1-\nu^{2}\right)}+\frac{16 a^{3} E\left(f_{u}\right)^{3}}{24\left(1-\nu^{2}\right)}+\frac{16 a^{3} E \cdot f_{l}\left(f_{l}+c\right)^{2}}{8}+\frac{16 a^{3} E \cdot f_{u}\left(f_{u}+c\right)^{2}}{8}+\frac{16 a^{3} \cdot E_{C} \cdot c^{3}}{12} \\
& X 24=\frac{20 a^{4} E\left(f_{l}\right)^{3}}{24\left(1-\nu^{2}\right)}+\frac{20 a^{4} E\left(f_{u}\right)^{3}}{24\left(1-\nu^{2}\right)}+\frac{20 a^{4} E \cdot f_{l}\left(f_{l}+c\right)^{2}}{8}+\frac{20 a^{4} E \cdot f_{u}\left(f_{u}+c\right)^{2}}{8}+\frac{20 a^{4} \cdot E_{C} \cdot c^{3}}{12} \\
& X 25=\frac{24 a^{5} E\left(f_{l}\right)^{3}}{24\left(1-\nu^{2}\right)}+\frac{24 a^{5} E\left(f_{u}\right)^{3}}{24\left(1-\nu^{2}\right)}+\frac{24 a^{5} E \cdot f_{l}\left(f_{l}+c\right)^{2}}{8}+\frac{24 a^{5} E \cdot f_{u}\left(f_{u}+c\right)^{2}}{8}+\frac{24 a^{5} \cdot E_{C} \cdot c^{3}}{12} \\
& Y 21=\frac{12 a^{2} E\left(f_{l}\right)^{3}}{24\left(1-\nu^{2}\right)}+\frac{12 a^{2} E\left(f_{u}\right)^{3}}{24\left(1-\nu^{2}\right)}+\frac{12 a^{2} E \cdot f_{l}\left(f_{l}+c\right)^{2}}{8}+\frac{12 a^{2} E \cdot f_{u}\left(f_{u}+c\right)^{2}}{8}+\frac{12 a^{2} \cdot E_{C} \cdot c^{3}}{12} \\
& Y 22=\frac{72 a c E^{2} \cdot f_{l}^{2}\left(f_{l}+c\right)^{2}}{8 \cdot G_{X Z}}+\frac{24 a^{3} E\left(f_{l}\right)^{3}}{24\left(1-\nu^{2}\right)}+\frac{24 a^{3} E\left(f_{u}\right)^{3}}{24\left(1-\nu^{2}\right)}+\frac{24 a^{3} E \cdot f_{l}\left(f_{l}+c\right)^{2}}{8}+\frac{24 a^{3} E \cdot f_{u}\left(f_{u}+c\right)^{2}}{8}+\frac{24 a^{3} \cdot E_{C} \cdot c^{3}}{12} \\
& Y 23=\frac{144 a^{2} c E^{2} \cdot f_{l}^{2}\left(f_{l}+c\right)^{2}}{8 \cdot G_{X Z}}+\frac{36 a^{4} E\left(f_{l}\right)^{3}}{24\left(1-\nu^{2}\right)}+\frac{36 a^{4} E\left(f_{u}\right)^{3}}{24\left(1-\nu^{2}\right)}+\frac{36 a^{4} E \cdot f_{l}\left(f_{l}+c\right)^{2}}{8}+\frac{36 a^{4} E \cdot f_{u}\left(f_{u}+c\right)^{2}}{8}+\frac{36 a^{4} \cdot E_{C} \cdot c^{3}}{12} \\
& Y 24=\frac{240 a^{3} c E^{2} \cdot f_{l}^{2}\left(f_{l}+c\right)^{2}}{8 \cdot G_{X Z}}+\frac{48 a^{5} E\left(f_{l}\right)^{3}}{24\left(1-\nu^{2}\right)}+\frac{48 a^{5} E\left(f_{u}\right)^{3}}{24\left(1-\nu^{2}\right)}+\frac{48 a^{5} E \cdot f_{l}\left(f_{l}+c\right)^{2}}{8}+\frac{48 a^{5} E \cdot f_{u}\left(f_{u}+c\right)^{2}}{8}+\frac{48 a^{5} \cdot E_{C} \cdot c^{3}}{12} \\
& Y 25=\frac{360 a^{4} c E^{2} \cdot f_{l}^{2}\left(f_{l}+c\right)^{2}}{8 \cdot G_{X Z}}+\frac{60 a^{6} E\left(f_{l}\right)^{3}}{24\left(1-\nu^{2}\right)}+\frac{60 a^{6} E\left(f_{u}\right)^{3}}{24\left(1-\nu^{2}\right)}+\frac{60 a^{6} E \cdot f_{l}\left(f_{l}+c\right)^{2}}{8}+\frac{60 a^{6} E \cdot f_{u}\left(f_{u}+c\right)^{2}}{8}+\frac{60 a^{6} \cdot E_{C} \cdot c^{3}}{12} \\
& Z 21=\frac{16 a^{3} E\left(f_{l}\right)^{3}}{24\left(1-\nu^{2}\right)}+\frac{16 a^{3} E\left(f_{u}\right)^{3}}{24\left(1-\nu^{2}\right)}+\frac{16 a^{3} E \cdot f_{l}\left(f_{l}+c\right)^{2}}{8}+\frac{16 a^{3} E \cdot f_{u}\left(f_{u}+c\right)^{2}}{8}+\frac{16 a^{3} \cdot E_{C} \cdot c^{3}}{12} \\
& Z 22=\frac{144 a^{2} c E^{2} \cdot f_{l}^{2}\left(f_{l}+c\right)^{2}}{8 \cdot G_{X Z}}+\frac{36 a^{4} E\left(f_{l}\right)^{3}}{24\left(1-\nu^{2}\right)}+\frac{36 a^{4} E\left(f_{u}\right)^{3}}{24\left(1-\nu^{2}\right)}+\frac{36 a^{4} E \cdot f_{l}\left(f_{l}+c\right)^{2}}{8}+\frac{36 a^{4} E \cdot f_{u}\left(f_{u}+c\right)^{2}}{8}+\frac{36 a^{4} \cdot E_{C} \cdot c^{3}}{12} \\
& Z 23=\frac{384 a^{3} c E^{2} \cdot f_{l}^{2}\left(f_{l}+c\right)^{2}}{8 \cdot G_{X Z}}+\frac{57.6 a^{5} E\left(f_{l}\right)^{3}}{24\left(1-\nu^{2}\right)}+\frac{57.6 a^{5} E\left(f_{u}\right)^{3}}{24\left(1-\nu^{2}\right)}+\frac{57.6 a^{5} E \cdot f_{l}\left(f_{l}+c\right)^{2}}{8}+\frac{57.6 a^{5} E \cdot f_{u}\left(f_{u}+c\right)^{2}}{8}+\frac{57.6 a^{5} \cdot E_{C} \cdot c^{3}}{12} \\
& Z 24=\frac{720 a^{4} c E^{2} \cdot f_{l}^{2}\left(f_{l}+c\right)^{2}}{8 \cdot G_{X Z}}+\frac{80 a^{6} E\left(f_{l}\right)^{3}}{24\left(1-\nu^{2}\right)}+\frac{80 a^{6} E\left(f_{u}\right)^{3}}{24\left(1-\nu^{2}\right)}+\frac{80 a^{6} E \cdot f_{l}\left(f_{l}+c\right)^{2}}{8}+\frac{80 a^{6} E \cdot f_{u}\left(f_{u}+c\right)^{2}}{8}+\frac{80 a^{6} \cdot E_{C} \cdot c^{3}}{12} \\
& Z 25=\frac{1152 a^{5} c E^{2} \cdot f_{l}^{2}\left(f_{l}+c\right)^{2}}{8 \cdot G_{X Z}}+\frac{720 a^{7} E\left(f_{l}\right)^{3}}{7 \cdot 24\left(1-\nu^{2}\right)}+\frac{720 a^{7} E\left(f_{u}\right)^{3}}{7 \cdot 24\left(1-\nu^{2}\right)}+\frac{720 a^{7} E \cdot f_{l}\left(f_{l}+c\right)^{2}}{56}+\frac{720 a^{7} E \cdot f_{u}\left(f_{u}+c\right)^{2}}{56}+\frac{720 a^{7} \cdot E_{C} \cdot c^{3}}{84} \\
& U 21=\frac{20 a^{4} E\left(f_{l}\right)^{3}}{24\left(1-\nu^{2}\right)}+\frac{20 a^{4} E\left(f_{u}\right)^{3}}{24\left(1-\nu^{2}\right)}+\frac{20 a^{4} E \cdot f_{l}\left(f_{l}+c\right)^{2}}{8}+\frac{20 a^{4} E \cdot f_{u}\left(f_{u}+c\right)^{2}}{8}+\frac{20 a^{4} \cdot E_{C} \cdot c^{3}}{12} \\
& U 22=\frac{240 a^{3} c E^{2} \cdot f_{l}^{2}\left(f_{l}+c\right)^{2}}{8 \cdot G_{X Z}}+\frac{48 a^{5} E\left(f_{l}\right)^{3}}{24\left(1-\nu^{2}\right)}+\frac{48 a^{5} E\left(f_{u}\right)^{3}}{24\left(1-\nu^{2}\right)}+\frac{48 a^{5} E \cdot f_{l}\left(f_{l}+c\right)^{2}}{8}+\frac{48 a^{5} E \cdot f_{u}\left(f_{u}+c\right)^{2}}{8}+\frac{48 a^{5} \cdot E_{C} \cdot c^{3}}{12} \\
& U 23=\frac{720 a^{4} c E^{2} \cdot f_{l}^{2}\left(f_{l}+c\right)^{2}}{8 \cdot G_{X Z}}+\frac{80 a^{6} E\left(f_{l}\right)^{3}}{24\left(1-\nu^{2}\right)}+\frac{80 a^{6} E\left(f_{u}\right)^{3}}{24\left(1-\nu^{2}\right)}+\frac{80 a^{6} E \cdot f_{l}\left(f_{l}+c\right)^{2}}{8}+\frac{80 a^{6} E \cdot f_{u}\left(f_{u}+c\right)^{2}}{8}+\frac{80 a^{6} \cdot E_{C} \cdot c^{3}}{12} \\
& U 24=\frac{1440 a^{5} c E^{2} \cdot f_{l}^{2}\left(f_{l}+c\right)^{2}}{8 \cdot G_{X Z}}+\frac{800 a^{7} E\left(f_{l}\right)^{3}}{7 \cdot 24\left(1-\nu^{2}\right)}+\frac{800 a^{7} E\left(f_{u}\right)^{3}}{7 \cdot 24\left(1-\nu^{2}\right)}+\frac{800 a^{7} E \cdot f_{l}\left(f_{l}+c\right)^{2}}{56}+\frac{800 a^{7} E \cdot f_{u}\left(f_{u}+c\right)^{2}}{56}+\frac{800 a^{7} \cdot E_{C} \cdot c^{3}}{84} \\
& U 25=\frac{2400 a^{6} c E^{2} \cdot f_{l}^{2}\left(f_{l}+c\right)^{2}}{8 \cdot G_{X Z}}+\frac{150 a^{8} E\left(f_{l}\right)^{3}}{24\left(1-\nu^{2}\right)}+\frac{150 a^{8} E\left(f_{u}\right)^{3}}{24\left(1-\nu^{2}\right)}+\frac{150 a^{8} E \cdot f_{l}\left(f_{l}+c\right)^{2}}{8}+\frac{150 a^{8} E \cdot f_{u}\left(f_{u}+c\right)^{2}}{8}+\frac{150 a^{8} \cdot E_{C} \cdot c^{3}}{12} \\
& V 21=\frac{24 a^{5} E\left(f_{l}\right)^{3}}{24\left(1-\nu^{2}\right)}+\frac{24 a^{5} E\left(f_{u}\right)^{3}}{24\left(1-\nu^{2}\right)}+\frac{24 a^{5} E \cdot f_{l}\left(f_{l}+c\right)^{2}}{8}+\frac{24 a^{5} E \cdot f_{u}\left(f_{u}+c\right)^{2}}{8}+\frac{24 a^{5} \cdot E_{C} \cdot c^{3}}{12} \\
& V 22=\frac{360 a^{4} c E^{2} \cdot f_{l}^{2}\left(f_{l}+c\right)^{2}}{8 \cdot G_{X Z}}+\frac{60 a^{6} E\left(f_{l}\right)^{3}}{24\left(1-\nu^{2}\right)}+\frac{60 a^{6} E\left(f_{u}\right)^{3}}{24\left(1-\nu^{2}\right)}+\frac{60 a^{6} E \cdot f_{l}\left(f_{l}+c\right)^{2}}{8}+\frac{60 a^{6} E \cdot f_{u}\left(f_{u}+c\right)^{2}}{8}+\frac{60 a^{6} \cdot E_{C} \cdot c^{3}}{12} \\
& V 23=\frac{1152 a^{5} c E^{2} \cdot f_{l}^{2}\left(f_{l}+c\right)^{2}}{8 \cdot G_{X Z}}+\frac{720 a^{7} E\left(f_{l}\right)^{3}}{7 \cdot 24\left(1-\nu^{2}\right)}+\frac{720 a^{7} E\left(f_{u}\right)^{3}}{7 \cdot 24\left(1-\nu^{2}\right)}+\frac{720 a^{7} E \cdot f_{l}\left(f_{l}+c\right)^{2}}{56}+\frac{720 a^{7} E \cdot f_{u}\left(f_{u}+c\right)^{2}}{56}+\frac{720 a^{7} \cdot E_{C} \cdot c^{3}}{84}
\end{aligned}
$$


$V 24=\frac{2400 a^{6} c E^{2} \cdot f_{l}^{2}\left(f_{l}+c\right)^{2}}{8 \cdot G_{X Z}}+\frac{150 a^{8} E\left(f_{l}\right)^{3}}{24\left(1-\nu^{2}\right)}+\frac{150 a^{8} E\left(f_{u}\right)^{3}}{24\left(1-\nu^{2}\right)}+\frac{150 a^{8} E \cdot f_{l}\left(f_{l}+c\right)^{2}}{8}+\frac{150 a^{8} E \cdot f_{u}\left(f_{u}+c\right)^{2}}{8}+\frac{150 a^{8} \cdot E_{C} \cdot c^{3}}{12}$
$V 25=\frac{28800 a^{7} c E^{2} \cdot f_{l}^{2}\left(f_{l}+c\right)^{2}}{56 \cdot G_{X Z}}+\frac{200 a^{9} E\left(f_{l}\right)^{3}}{24\left(1-\nu^{2}\right)}+\frac{200 a^{9} E\left(f_{u}\right)^{3}}{24\left(1-\nu^{2}\right)}+\frac{200 a^{9} E \cdot f_{l}\left(f_{l}+c\right)^{2}}{8}+\frac{200 a^{9} E \cdot f_{u}\left(f_{u}+c\right)^{2}}{8}+\frac{200 a^{9} \cdot E_{C} \cdot c^{3}}{12}$

$$
\begin{aligned}
& k_{11}=\frac{2}{5} a^{5}+\left(c+f_{u}+f_{l}\right) \cdot \frac{8}{5} a^{5}+\left(\left(f_{l}\right)^{3}+\left(f_{u}+c\right)^{3}\right) \cdot \frac{8}{9} a^{3} \\
& k_{12}=\frac{1}{3} a^{6}+\left(c+f_{u}+f_{l}\right) \cdot 2 a^{6}+\left(\left(f_{l}\right)^{3}+\left(f_{u}+c\right)^{3}\right) \cdot a^{4} \\
& k_{13}=\frac{2}{7} a^{7}+\left(c+f_{u}+f_{l}\right) \cdot \frac{16}{7} a^{7}+\left(\left(f_{l}\right)^{3}+\left(f_{u}+c\right)^{3}\right) \cdot \frac{16}{15} a^{5} \\
& k_{14}=\frac{2}{8} a^{8}+\left(c+f_{u}+f_{l}\right) \cdot \frac{20}{8} a^{8}+\left(\left(f_{l}\right)^{3}+\left(f_{u}+c\right)^{3}\right) \cdot \frac{10}{9} a^{6} \\
& k_{15}=\frac{2}{9} a^{9}+\left(c+f_{u}+f_{l}\right) \cdot \frac{24}{9} a^{9}+\left(\left(f_{l}\right)^{3}+\left(f_{u}+c\right)^{3}\right) \cdot \frac{24}{21} a^{7} \\
& k_{21}=\frac{1}{3} a^{6}+\left(c+f_{u}+f_{l}\right) \cdot 2 a^{6}+\left(\left(f_{l}\right)^{3}+\left(f_{u}+c\right)^{3}\right) \cdot a^{4} \\
& k_{22}=\frac{2}{7} a^{7}+\left(c+f_{u}+f_{l}\right) \cdot \frac{18}{7} a^{7}+\left(\left(f_{l}\right)^{3}+\left(f_{u}+c\right)^{3}\right) \cdot \frac{18}{15} a^{5} \\
& k_{23}=\frac{2}{8} a^{8}+\left(c+f_{u}+f_{l}\right) \cdot \frac{24}{8} a^{8}+\left(\left(f_{l}\right)^{3}+\left(f_{u}+c\right)^{3}\right) \cdot \frac{8}{6} a^{6} \\
& k_{24}=\frac{2}{9} a^{9}+\left(c+f_{u}+f_{l}\right) \cdot \frac{30}{9} a^{9}+\left(\left(f_{l}\right)^{3}+\left(f_{u}+c\right)^{3}\right) \cdot \frac{10}{7} a^{7} \\
& k_{25}=\frac{2}{10} a^{10}+\left(c+f_{u}+f_{l}\right) \cdot \frac{36}{10} a^{10}+\left(\left(f_{l}\right)^{3}+\left(f_{u}+c\right)^{3}\right) \cdot \frac{3}{2} a^{8} \\
& k_{31}=\frac{2}{7} a^{7}+\left(c+f_{u}+f_{l}\right) \cdot \frac{16}{7} a^{7}+\left(\left(f_{l}\right)^{3}+\left(f_{u}+c\right)^{3}\right) \cdot \frac{16}{15} a^{5} \\
& k_{32}=\frac{2}{8} a^{8}+\left(c+f_{u}+f_{l}\right) \cdot \frac{24}{8} a^{8}+\left(\left(f_{l}\right)^{3}+\left(f_{u}+c\right)^{3}\right) \cdot \frac{8}{6} a^{6} \\
& k_{33}=\frac{2}{9} a^{9}+\left(c+f_{u}+f_{l}\right) \cdot \frac{32}{9} a^{9}+\left(\left(f_{l}\right)^{3}+\left(f_{u}+c\right)^{3}\right) \cdot \frac{32}{21} a^{7} \\
& k_{34}=\frac{2}{10} a^{10}+\left(c+f_{u}+f_{l}\right) \cdot \frac{40}{10} a^{10}+\left(\left(f_{l}\right)^{3}+\left(f_{u}+c\right)^{3}\right) \cdot \frac{5}{3} a^{8} \\
& k_{35}=\frac{2}{11} a^{11}+\left(c+f_{u}+f_{l}\right) \cdot \frac{48}{11} a^{11}+\left(\left(f_{l}\right)^{3}+\left(f_{u}+c\right)^{3}\right) \cdot \frac{48}{27} a^{9} \\
& k_{41}=\frac{2}{8} a^{8}+\left(c+f_{u}+f_{l}\right) \cdot \frac{20}{8} a^{8}+\left(\left(f_{l}\right)^{3}+\left(f_{u}+c\right)^{3}\right) \cdot \frac{10}{9} a^{6} \\
& k_{42}=\frac{2}{9} a^{9}+\left(c+f_{u}+f_{l}\right) \cdot \frac{30}{9} a^{9}+\left(\left(f_{l}\right)^{3}+\left(f_{u}+c\right)^{3}\right) \cdot \frac{30}{21} a^{7} \\
& k_{43}=\frac{2}{10} a^{10}+\left(c+f_{u}+f_{l}\right) \cdot \frac{40}{10} a^{10}+\left(\left(f_{l}\right)^{3}+\left(f_{u}+c\right)^{3}\right) \cdot \frac{5}{3} a^{8} \\
& k_{44}=\frac{2}{11} a^{11}+\left(c+f_{u}+f_{l}\right) \cdot \frac{50}{11} a^{11}+\left(\left(f_{l}\right)^{3}+\left(f_{u}+c\right)^{3}\right) \cdot \frac{50}{27} a^{9} \\
& k_{45}=\frac{2}{12} a^{12}+\left(c+f_{u}+f_{l}\right) \cdot \frac{60}{12} a^{12}+\left(\left(f_{l}\right)^{3}+\left(f_{u}+c\right)^{3}\right) \cdot 2 a^{10} \\
& k_{51}=\frac{2}{9} a^{9}+\left(c+f_{u}+f_{l}\right) \cdot \frac{24}{9} a^{9}+\left(\left(f_{l}\right)^{3}+\left(f_{u}+c\right)^{3}\right) \cdot \frac{24}{21} a^{7} \\
& k_{52}=\frac{2}{10} a^{10}+\left(c+f_{u}+f_{l}\right) \cdot \frac{36}{10} a^{10}+\left(\left(f_{l}\right)^{3}+\left(f_{u}+c\right)^{3}\right) \cdot \frac{36}{24} a^{8} \\
& k_{53}=\frac{2}{11} a^{11}+\left(c+f_{u}+f_{l}\right) \cdot \frac{48}{11} a^{11}+\left(\left(f_{l}\right)^{3}+\left(f_{u}+c\right)^{3}\right) \cdot \frac{48}{27} a^{9} \\
& k_{54}=\frac{2}{12} a^{12}+\left(c+f_{u}+f_{l}\right) \cdot \frac{60}{12} a^{12}+\left(\left(f_{l}\right)^{3}+\left(f_{u}+c\right)^{3}\right) \cdot 2 a^{10} \\
& k_{55}=\frac{2}{13} a^{13}+\left(c+f_{u}+f_{l}\right) \cdot \frac{72}{13} a^{13}+\left(\left(f_{l}\right)^{3}+\left(f_{u}+c\right)^{3}\right) \cdot \frac{72}{33} a^{11}
\end{aligned}
$$




\section{References}

[1] R.D. Mindlin, Influence of rotatory inertia and shear on flexural motions of isotropic elastic plates, J. Appl. Mech. (1951) 1831-1838.

[2] S. Yarlagadda, G. Lesieutre, Fiber contribution to modal damping of polymer matrix composite panels, J. Spacecraft Rockets 32 (1995) 825-831

[3] C.M. Wang, Deducing thick plate solutions from classical thin plate solutions, Struct. Eng. Mech. 11 (2001) 89-104

[4] M. Meunier, R.A Shenoi, Dynamic analysis of composite sandwich plates with damping modeled using higherorder shear deformation theory, Compos. Struct. 54 (2001) 243-254

[5] M. Soula, R. Nasri, M.A. Ghazel, Y. Chevalier, The effects of kinematic model approximations on natural frequencies and modal damping of laminated composite plates, J. Sound Vib. 297 (2006) 315-328

[6] M.R. Maheri, R.D. Adams, Steady-state flexural vibration damping of honeycomb sandwich beams, Compos. Sci. Technol. 52 (1994) 333-347
[7] J.R. Banerjee, Frequency equation and modal deformation formulae for composite Timoshenko beams, Compos. Struct. 51 (2001) 381-388

[8] E. Nilsson, A.C. Nilsson, Prediction and Measurement of some dynamic properties of sandwich structures with honeycomb and foam cores, J. Sound Vib. 251 (2002) 409-430

[9] R.D. Blevins, Formulas for Natural Frequency and Mode Shape,Van Nostrand and Reinhold compagny, Litton Educational Publishing Inc, Copyright (C) (1979) : ISBN: 0-442-20710-7 (252 pages), pp. 108-113

[10] E. Fotsing, M. Sola, A. Ross, E. Ruiz, Lightweight damping of composite sandwich beams: Experimental analysis, J. Compos. Mater. 47 (2012) 1501-1511

[11] G.R. Rayjade, G.V.R. Seshagiri Rao, Study of composite sandwich structure and bending characteristics, Int. J. Curr. Eng. Technol. 5 (2015) 797-802

[12] N.A. Rubayi, S. Charoenree, Natural frequencies of vibration of cantilever sandwich beams, Comput. Struct. 6 (1976) 345-353 